pathway and may be an extracolonic manifestation of familial adenomatous polyposis. Am J Surg Pathol 2018; 42: 137-9.

7. Irving JA, Lee $\mathrm{C}-\mathrm{H}$, Yip $\mathrm{S}$, et al. Microcystic stromal tumor: a distinctive ovarian sex cord-stromal neoplasm characterized by FOXL2 SF-1, WT-1, Cyclin D1, and beta-catenin nuclear expression and CTNNB1 mutations. Am J Surg Pathol 2015; 39: 1420-6.

8. Rabban JT, Karnezis AN, Devine WP. Practical roles for molecular diagnostic testing in ovarian adult granulosa cell tumour, Sertoli-Leydig cell tumour, microcystic stromal tumour and their mimics. Histopathology 2020; 76: 11-24.

9. Deshpande V, Oliva E, Young RH. Solid pseudopapillary neoplasm of the ovary: a report of 3 primary ovarian tumors resembling those of the pancreas. Am J Surg Pathol 2010; 34: 1514-20.

10. Komforti MK, Edelman M, Fan C, Liang SX. Solid pseudopapillary neoplasm presenting as a primary ovarian mass in an eighteen-year-old female: report of a case and review of the literature. Virchows Arch 2018; 472: 285-91.

11. McKenna M, Kenny B, Dorman G, McCluggage WG. Combined adult granulosa cell tumor and mucinous cystadenoma of the ovary: granulosa cell tumor with heterologous mucinous elements. Int J Gynecol Pathol 2005; 24: 224-7.

12. Zhang Y, Tao L, Yin C, et al. Ovarian microcystic stromal tumor with undetermined potential: case study with molecular analysis and literature review. Hum Pathol 2018; 78: 171-6.

DOI: https://doi.org/10.1016/j.pathol.2021.02.018

\section{Infectious crystalline keratopathy: the clinical utility of periodic acid- Schiff stain}

Sir,

Infectious crystalline keratopathy (ICK) describes a pattern of chronic corneal infection characterised by needle-shaped colonies of microorganisms growing between collagen lamella with disproportionally little inflammation. ${ }^{1}$ The condition arises when microorganisms gain access to the corneal stroma of patients whose local immune response has been suppressed by topical corticosteroids. The majority of infections are caused by low virulence bacteria, but fungi and acanthamoeba have been reported. ${ }^{1}$ We describe a patient with ICK due to periodic acid-Schiff (PAS)-positive bacteria who developed post-operative endophthalmitis more than a month after penetrating keratoplasty. Delayed onset postoperative endophthalmitis is an uncommon but serious complication of intraocular surgery. Knowledge that PASpositive bacteria are putative contaminants in this clinical setting can alter management.

A man in his late eighties underwent a penetrating keratoplasty to restore vision that had declined to 'hand motions' from a failed corneal transplant (Descemet's stripping endothelial automated keratoplasty) 6 years earlier for Fuchs' dystrophy. For 9 months prior to penetrating keratoplasty the patient was treated with topical prednisolone acetate $1 \%$ (varying from hourly to four times per day). During this time the cornea was diffusely oedematous and focally 'cloudy'. Surgery was uncomplicated; no tissue was submitted for culture. The clinicians were alerted days after surgery that the cornea was infected (see below). Topical moxifloxacin, started after surgery, was discontinued on post-operative day 22. Recovery remained uneventful until post-operative day 42 when the patient developed anterior chamber inflammation and elevated intraocular pressure. He was started on topical prednisolone acetate $1 \%$ for non-specific 'uveitis' and pressure lowering medications (dorzolamide 2\%/timolol $0.5 \%$ ). On post-operative day 50, inflammation substantially worsened so oral valtrex was begun on the presumptive diagnosis of herpes uveitis (there was no previous history of ocular herpes infection or any characteristic findings, e.g., epithelial dendrite, etc.). Inflammation worsened until post-operative day 93 at which time cultures from aqueous and vitreous humours were taken; both grew Corynebacterium striatum with greater colony counts from the anterior chamber. Intravitreous antibiotics (ceftazidime $2.25 \mathrm{mg} / 0.1 \mathrm{~mL}$; vancomycin $1 \mathrm{mg} / 0.1 \mathrm{~mL}$ ) were injected the day the samples were obtained; intraocular inflammation progressively improved over several weeks. Final visual acuity was $20 / 400$.

On microscopic examination, the central cornea was superficially ulcerated. Innumerable bacteria were growing in stroma between collagen lamellae forming spindle shaped colonies (Fig. 1). There was modest focal acute inflammation around the outer edge of bacterial colonies. Deeper stroma and Descemet's membrane (earlier graft tissue) were separated from anterior stroma. Descemet's membrane was covered on its posterior surface with fibrin and degenerating neutrophils Gram stain showed mostly Gram-positive cocci and small nonbranching Gram-positive rods (Fig. 1C). The bacteria stained strongly with PAS after diastase and were acid-fast negative with Ziehl-Neelsen and Fite stains. A Gomori methenamine silver (GMS) stain was negative for fungus.

The histopathological findings in this case were diagnostic of ICK, namely the biofilm-like proliferation of bacteria between collagen lamellae with disproportionately little inflammatory response. Secondary endophthalmitis is an anticipated complication after contaminated intraocular surgery, but in this case endophthalmitis was unusual in that the signs of intraocular inflammation were delayed more than a month after surgery. ${ }^{2,3}$ Delayed onset endophthalmitis is a manifestation of a limited subset of low-virulence organisms. The putative bacterial contaminants found in this case were PAS-positive which also limits possible pathogens. PASpositive bacteria include Bacillus cereus, Klebsiella pneunomiae, Micrococcus luteus, Propionibacterium, Corynebacterium, Tropheryma whipplei, Actinomyces, and atypical mycobacteria. ${ }^{4,5}$ In context of this case, GMS and AFB stains along with morphological findings excluded atypical mycobacteria and actinomycetes. Bacillus cereus and Klebsiella pneumonia are highly virulent ocular pathogens and cause rapidly progressive endophthalmitis. ${ }^{6}$ Micrococcus luteus and Tropheryma whipplei have not been implicated in postoperative endophthalmitis. To our knowledge, only two PAS-positive bacteria have been associated with chronic (e.g., delayed onset) endophthalmitis: propionibacterium and corynebacterium. ${ }^{7,8}$

Corynebacterium spp., are Gram-positive bacilli that are part of the normal flora of the skin and eyelid. Most members of this genus are regarded as laboratory contaminants that rarely cause human infection. Corynebacterium striatum, however, may be different. It has been implicated in cases of endocarditis, meningitis, bacteraemia, and respiratory infection. ${ }^{9,10}$ In the context of this case, $C$. striatum appears as both a low grade pathogen and an opportunistic organism that has colonised corneal stroma. The patient had no systemic immune-compromising conditions (other than age) but the structural integrity of his eye was compromised by the complications of past surgery. 


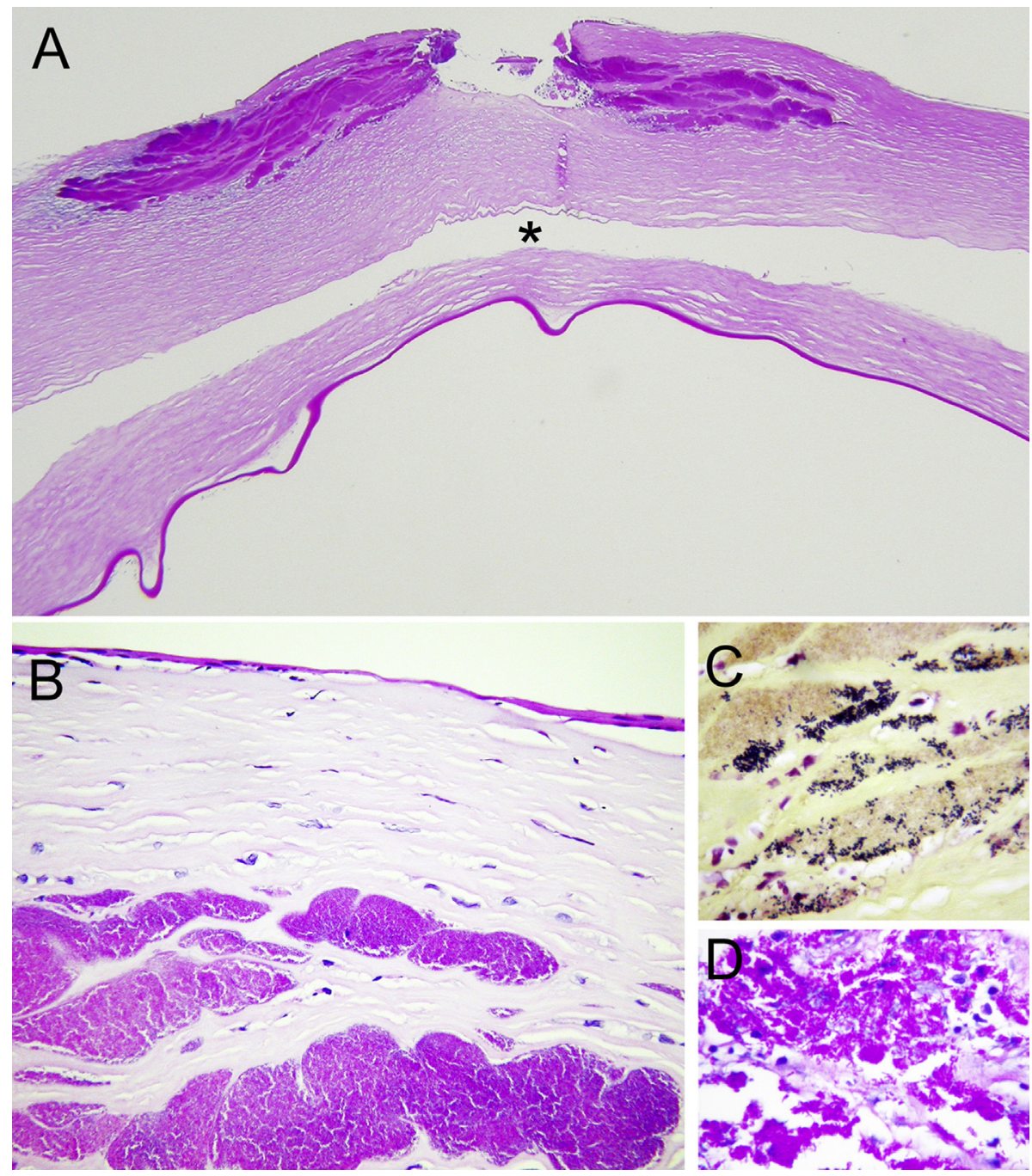

Fig. 1 (A) Removed corneal button with anterior stroma filled with PAS-positive bacteria and relatively minimal inflammation. The space between anterior threefourths stroma $\left(^{*}\right)$ and posterior fourth represents the non-union of host and donor tissues from transplant performed 6 years earlier. (B) Higher magnification showing myriad PAS-positive bacteria in near absence of inflammatory cells. (C) Gram stains showing a minority of bacteria as Gram-positive cocci and some smal rods. (D) PAS stain at edge of colony showing small cocci and small rod-shaped organisms. The density and intensity of staining precluded good morphological detail of bacteria.

Unlike most cases of post-operative bacterial endophthalmitis that show signs of intraocular inflammation within days to a week of surgery, several low virulence organisms can result in delayed clinical manifestations. In the setting of contaminated surgery from PAS-positive bacteria, the exclusion of actinomyces and atypical mycobacteria by GMS and AFB stains and morphology narrows the possible causes of post-operative infection to high and low virulent bacteria Intraocular infections with $B$. cereus and $K$. pneumoniae have rapidly progressive clinical courses often resulting in loss of the eye within days. ${ }^{6}$ Knowledge that a potential bacterial contaminant of intraocular surgery is PAS-positive provides useful information by narrowing the list of possible pathogens. That list includes several capable of causing delayed onset endophthalmitis.

Conflicts of interest and sources of funding: The authors state that there are no conflicts of interest to disclose.

\section{Curtis E. Margo ${ }^{1}$, Jean Guffey Johnson ${ }^{2}$}

${ }^{1}$ Department of Pathology and Cell Biology, and Ophthalmology, Morsani College of Medicine, Tampa, USA; ${ }^{2}$ Department of Pathology, James A Haley Veterans Hospital, Tampa, USA

Contact Dr Curtis E. Margo.

E-mail: cmargo@usf.edu

1. Porter AJ, Lee G, Jun AS. Infectious crystalline keratopathy. Surv Ophthalmol 2017; 63: 480-99.

2. Uy HS, Nguyen QD, Durand ML, et al. Infectious crystalline keratopathy and endophthalmitis secondary to Mycobacterium abscessus in a monocular patient with Steven-Johnson syndrome. Am J Ophthalmol 1999; 127: 209-10.

3. Garagania SP, Mela EK, Karamanos NK, et al. Infectious crystalline keratopathy and endophthalmitis caused by a slime producing Staphylococcus epidermidis strain. Ann Ophthalmol 2004 36: $37-90$ 
4. Khavari PA, Bolognia JL, Eisen R, et al. Periodic acid-Schiff-positive organisms in primary cutaneous Bacillus cereus infection. Case report and an investigation of the periodic acid-Schiff staining properties of bacteria. Arch Dermatol 1991; 127: 543-56.

5. Csillag A. Periodic acid-Schiff (PAS) staining of 'atypical' mycobacteria and tubercle bacilli. Tubercle 1960; 41: 63-7.

6. Margo CE. Bacterial infections. In: Klintworth GK, Garner A, editors Pathobiology of Ocular Disease. 3rd ed. New York: Informa, 2008; $213-34$

7. Margo CE, Pavan PR, Groden L. Chronic vitritis with macrophage inclusions. A sequela of treated endophthalmitis due to a Corynebacterium. Ophthalmology 1988; 95: 156-61.
8. Clark WL, Kaiser PK, Flynn HW, et al. Treatment strategies and visual acuity outcomes in chronic postoperative Propionibacterium acnes endophthalmitis. Ophthalmology 1999; 106: 1665-70.

9. Shariff M, Aditi Beri K. Corynebacterium striatum: an emerging respiratory pathogen. J Infect Dev Ctries 2018; 12: 581-6.

10. McMullen AR, Anderson N, Wallace MA, et al. When good bugs go bad: epidemiology of antimicrobial resistance profiles of Corynebacterium striatum, an emerging multidrug-resistant, opportunistic pathogen. Antimicrob Agents Chemother 2017; 61: e01111-7.

DOI: https://doi.org/10.1016/j.pathol.2021.01.015 